Boise State University

ScholarWorks

$2-1-2013$

\title{
Imaging Stability in Force-Feedback High-Speed Atomic Force Microscopy
}

Byung I. Kim

Boise State University

Ryan Boehm

Boise State University 
NOTICE: this is the author's version of a work that was accepted for publication in Ultramicroscopy. Changes resulting from the publishing process, such as peer review, editing, corrections, structural formatting, and other quality control mechanisms may not be reflected in this

document. Changes may have been made to this work since it was submitted for publication. A definitive version was subsequently published in Ultramicroscopy, 2012. DOI: 10.1016/j.ultramic.2012.09.012

\title{
Imaging Stability in Force-Feedback High-Speed Atomic Force Microscopy
}

\author{
Byung I. Kim and Ryan D. Boehm \\ Department of Physics, Boise State University \\ 1910 University Drive, Boise, ID 83725-1570 \\ United States of America \\ Email: ByungKim@boisestate.edu
}

\begin{abstract}
We studied the stability of force-feedback high-speed atomic force microscopy (HSAFM) by imaging soft, hard, and biological sample surfaces at various applied forces. The HSAFM images showed sudden topographic variations of streaky fringes with a negative applied force when collected on a soft hydrocarbon film grown on a grating sample, whereas they showed stable topographic features with positive applied forces. The instability of HSAFM images with the negative applied force was explained by the transition between contact and noncontact regimes in the force-distance curve. When the grating surface was cleaned, and thus hydrophilic by removing the hydrocarbon film, enhanced imaging stability was observed at both positive and negative applied forces. The higher adhesive interaction between the tip and the surface explains the improved imaging stability. The effects of imaging rate on the imaging stability were tested on an even softer adhesive Escherichia coli biofilm deposited onto the grating structure. The biofilm and planktonic cell structures in HSAFM images were reproducible within the force deviation less than $\sim 0.5 \mathrm{nN}$ at the imaging rate up to $0.2 \mathrm{~s}$ per frame, suggesting that the force-feedback HSAFM was stable for various imaging speeds in imaging softer adhesive biological samples.
\end{abstract}

Keywords: COIFM; AFM; HSAFM; interfacial force spectroscopy; force-feedback; snap-to-contact; imaging stability. 
NOTICE: this is the author's version of a work that was accepted for publication in Ultramicroscopy. Changes resulting from the publishing process, such as peer review, editing, corrections, structural formatting, and other quality control mechanisms may not be reflected in this document. Changes may have been made to this work since it was submitted for publication. A definitive version was subsequently published in Ultramicroscopy, 2012. DOI: 10.1016/j.ultramic.2012.09.012

\section{Introduction}

Most widely used atomic force microscopy (AFM) techniques have a limited ability to detect the static force at a few nanometers above the attractive surface due to mechanical instabilities of the tip-sensor assembly called the "snap-to-contact" phenomenon [1-4]. This phenomenon corresponds to the sudden transition from the noncontact to contact states in contact mode AFM [4]. The transition is caused by the uncontrolled movement of the probe to the surface when the intermolecular force gradient $\left(\partial F_{i} / \partial z\right)$ exceeds the spring constant of the force probe $(k)$. The contact mode AFM is unable to image topographic structures of the surface in the noncontact regime due to this mechanical instability.

To avoid the snap-to-contact phenomenon, amplitude modulation (AM) AFM was introduced to image soft samples or to visualize electric or magnetic structures, which generate long-ranged electric or magnetic forces. Kühle et al. found that several points of instability in the parameter space caused by attractive forces combined with repulsive elastic-type forces resulted in disturbances during image acquisition on hard elastic surfaces [5]. The AM-AFM images generated some protruded artifacts when the probe encountered the high hillock structures. These features were interpreted as nonlinear stochastic phenomena in the tip-sample interactions [6]. The abrupt changes in the height of topographic features were associated with the continual switching of the oscillating tip between the two stable states while the tip scans the surface in the AM-AFM [6-8]. Similarly, the topographic features in the magnetic image are attributed to the switching between the bistable states in magnetic force microscopy (MFM) [9]. This concept has been applied to separate the topographic structure from magnetic images using electrostatic modulation. In principle, both effects of the AM-AFM and MFM result from the intrinsic nonlinear mechanical bistability of the sensor-sample assembly.

As an alternative approach, the snap-to-contact issue associated with contact AFM has been resolved by removing the bistability using voltage-activated force feedback $[10,11]$. Previously, we developed an experimental technique called "cantilever-based optical interfacial microscopy" (COIFM) to measure 
NOTICE: this is the author's version of a work that was accepted for publication in Ultramicroscopy. Changes resulting from the publishing process, such as peer review, editing, corrections, structural formatting, and other quality control mechanisms may not be reflected in this document. Changes may have been made to this work since it was submitted for publication. A definitive version was subsequently published in Ultramicroscopy, 2012. DOI: 10.1016/j.ultramic.2012.09.012

fundamental interfacial interactions at nanometer scales [11-13]. The COIFM utilizes a force-feedback technique to avoid the rapid snap-to-contact process associated with AFM measurement. By employing a feedback scheme, the spring constant can be considered infinite due to the counter force. It has proven its ability to simultaneously measure normal and friction forces between two surfaces at the nanometer scale in ambient environments using lateral modulation $[12,13]$. The COIFM was later adapted for high-speed AFM (HSAFM) imaging of large-scale biological specimens through the force-feedback scheme [14]. However, contrary to the AM-AFM, there have been few studies on how the stability influences the imaging in the force-feedback HSAFM. We investigated this effect by comparing both interfacial force spectroscopic and topographic information.

\section{Materials and methods}

We used a force-feedback HSAFM that had been previously developed and reported [14] by utilizing a commercially available cantilever called a "dimensional micro-actuated silicon probe" (DMASP) as the sensor and actuator. The actuation function of the DMASP was used to maintain the constant deflection of the cantilever at an applied force during the data acquisition through the application of output voltage from the force-feedback controller. The output voltage was converted into the applied force by multiplying the conversion factor $(5 \mathrm{nN} / \mathrm{V})$ reported in our earlier publication [14]. The voltage applied to the piezo tube was recorded as a topographic signal for each AFM image displayed with $128 \times 128$ data points.

All data presented here were recorded through analog-to-digital converter (ADC) inputs of the RHK 100 and XPM PRO software. For the collection of force-distance curves, the force-feedback voltage $\left(\mathrm{V}_{\mathrm{ZnO}}\right)$ was recorded as a function of distance between the probe and the sample surface during both approach and retraction of the piezo tube $[11,13]$. We obtained force-distance curves under the off-feedback condition by sweeping the computer-controlled input of a $\mathrm{z}$ high-voltage amplifier.

All data were collected on an oxidized silicon two-dimensional grating sample with $10 \mu \mathrm{m}$ periodicity (laterally) and $180 \mathrm{~nm}$ step height acquired from Veeco, Inc. [15] at a relative humidity of 30\% at a room 
NOTICE: this is the author's version of a work that was accepted for publication in Ultramicroscopy. Changes resulting from the publishing process, such as peer review, editing, corrections, structural formatting, and other quality control mechanisms may not be reflected in this document. Changes may have been made to this work since it was submitted for publication. A definitive version was subsequently published in Ultramicroscopy, 2012. DOI: 10.1016/j.ultramic.2012.09.012

temperature of $22^{\circ} \mathrm{C}$. A soft hydrocarbon film was grown on the grating sample, which had been exposed to the ambient environment for 60 days. The ambient material allows for the covering of the hydrophilic grating sample with a layer consisting of hydrophobic properties [16]. Topographic and force images were taken with alternating positive and negative applied forces. After data acquisition the grating sample underwent a cleaning procedure consisting of submersion into a piranha solution made from a 3:1 concentrated $\mathrm{H}_{2} \mathrm{SO}_{4} / 30 \% \mathrm{H}_{2} \mathrm{O}_{2}$ (Pharmco and Fischer Scientific, respectively); then sonicated in acetone, ethanol, and distilled water for $5 \mathrm{~min}$ in each; and ultimately dried with an $\mathrm{N}_{2}$ flow. This allowed for comparison and correlation of the effects of hydrophilicity. To demonstrate its applicability to biological samples, an Escherichia coli (E. coli) culture and biofilm sample was taken from a PBS solution and deposited onto the clean grating structure. The culture of a non-pathogenic strand of E. coli (RK4353) was grown overnight with shaking $(225 \mathrm{rpm})$ at $37^{\circ} \mathrm{C}$ in $5 \mathrm{~mL}$ of Luria Bertani broth. The culture was pelleted at $6000 \mathrm{xg} / 10 \mathrm{~min}$, and resuspended in sterile PBS. $1 \mathrm{~mL}$ of the mixture was then dispensed into $99 \mathrm{~mL}$ of fresh PBS solution, causing a 1:100 part dilution. The $10 \mu \mathrm{m}$ periodicity and $200 \mathrm{~nm}$ step height of the grating sample were used for an additional dimensional scale for each collected image. Topographic and force images were taken at the applied force of $0 \mathrm{nN}$ for various scan speeds on the biofilm and E. coli samples.

\section{Results and discussion}

The force-feedback HSAFM was applied to high-speed imaging on a soft hydrocarbon film with large topographic height variations to investigate the stability of the system. First, force-distance curves were collected on the grating sample covered by the adsorbed material film. As expected both for the approach Fig. 1(a) and retraction Fig. 1(b) curves do not show any snap-to-contact phenomenon due to the zero compliance effect during the force-distance measurement. The data show that the minimum force is -1.5 $\mathrm{nN}$ on approach, while the minimum force is $-2 \mathrm{nN}$ on retraction. The displayed minute attractive force encountered on approach (as seen in Fig. 1(a)) implies that the outer surface of the adsorbed material is hydrophobic in nature, consistent with our earlier report [16]. The hydrophobicity of the surfaces is 
NOTICE: this is the author's version of a work that was accepted for publication in Ultramicroscopy. Changes resulting from the publishing process, such as peer review, editing, corrections, structural formatting, and other quality control mechanisms may not be reflected in this document. Changes may have been made to this work since it was submitted for publication. A definitive version was subsequently published in Ultramicroscopy, 2012. DOI: 10.1016/j.ultramic.2012.09.012

derived from the nonpolar hydrophobic surface, which prefers to orient outward to be exposed to ambient air molecules which are predominantly non-polar. The stiffness of the sample was found to be $\sim 0.77 \mathrm{~N} / \mathrm{m}$ from a linear curve fitting made in the repulsive regime from $-5 \mathrm{~nm}$ to $2 \mathrm{~nm}$ tip-sample distance in Fig. 1(b).
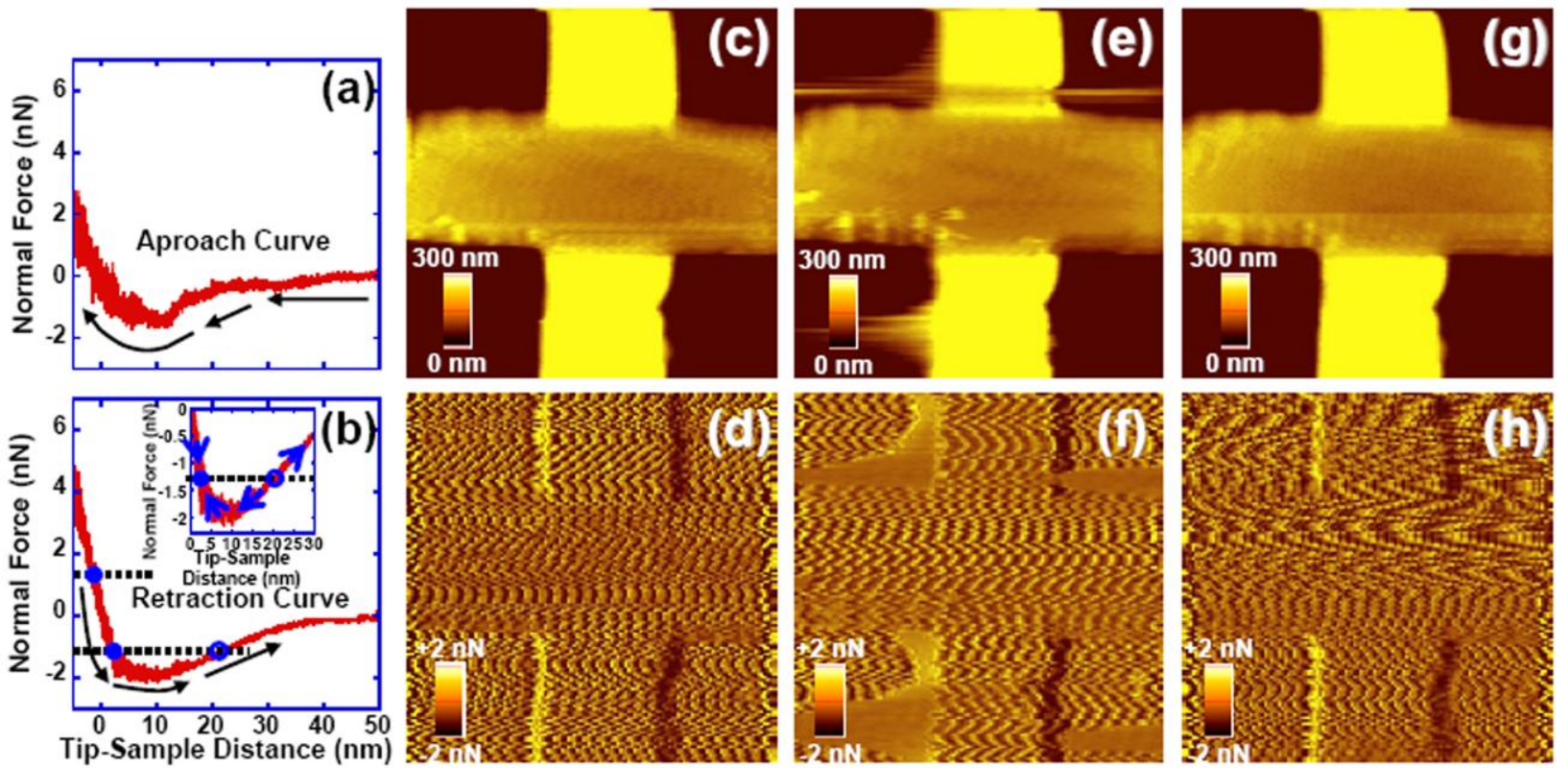

Fig. 1. Force-distance curve between the tip and a two-dimensional grating structure with $10 \mu \mathrm{m}$ periodicity, with a force activated voltage feedback system during approach (a) and retraction (b). Dashed lines represent the imaging applied force values, where the closed circles represent intersection of the curve with negative slope and the open circle represent intersection of the curve with positive slope. Inset is a zoomed in view of the force distance curve at the negative-positive slope transition point. The addition of the arrows in the inset indicate the direction the feedback moves after moving past one of the imaging states indicated by the closed and open circle on the dashed line symbolizing the applied force. Constant-force topographic $(\mathbf{c}, \mathbf{e}, \mathbf{g})$ and simultaneous force (d,f,h) images collected in a scan size of $10 \mu \mathrm{m} \times 10 \mu \mathrm{m}$ on the grating structure at an imaging rate of $10 \mathrm{~s}$ per frame at applied forces of $+1.25 \mathrm{nN}(\mathbf{a}, \mathbf{b}),-1.25 \mathrm{nN}(\mathbf{c}, \mathbf{d}),+1.25 \mathrm{nN}(\mathbf{e , f})$.

As seen in Fig. 1(b), when imaging with a positive applied force (indicated by a dotted line representing the applied force of $+1.25 \mathrm{nN}$ ), there is only one stable imaging condition (the intersection of the dotted line and the force-distance curve, represented by a solid circle). However, when imaging with a negative applied force, there are one stable and one unstable imaging conditions indicated by the solid circle and open circle, respectively, on the dotted line at $-1.25 \mathrm{nN}$. We hypothesized that when imaging the grating sample, as the tip reached the sudden height deviation associated with the step structure, the tip-sample distance would momentarily increase, which would cause a negative rise in force within the force- 
NOTICE: this is the author's version of a work that was accepted for publication in Ultramicroscopy. Changes resulting from the publishing process, such as peer review, editing, corrections, structural formatting, and other quality control mechanisms may not be reflected in this document. Changes may have been made to this work since it was submitted for publication. A definitive version was subsequently published in Ultramicroscopy, 2012. DOI: 10.1016/j.ultramic.2012.09.012

distance curve. This negative rise could potentially trigger a shift from the negative sloped stable imaging condition (closed circle) to the positive sloped unstable imaging condition (open circle), thus causing imaging instability as the tip transitioned between the two imaging states. When encountering a large topographic variation, the tip-sample distance change will cause the feedback of the system to adjust the tip-sample distance in such a manner as it perceives will bring the system back to the constant-force imaging condition (as depicted by the inset of Fig. 1(b)). However, if the system is pushed past the unstable imaging point (open circle), the system will reset and attempt to increase the tip-sample distance in order to increase force as if it were near the stable imaging condition. This causes the feedback to move in the wrong direction (as in the direction of the right-most arrow), creating an unstable imaging condition. At the applied force of $-1.25 \mathrm{nN}$, because only a slight amount of force $\sim 0.75 \mathrm{nN}$ is needed to make the transition between the two states, the feedback perturbation (i.e. the sudden tip-sample distance change) should allow for switching between the imaging states at almost equal probability during the data acquisition near the step-structures of the grating sample.

To test this hypothesis, a sequence of high-speed imaging with different loading forces was performed.

Fig. 1(c) and 1(d) show topographic and force images taken at a high-speed imaging rate of $10 \mathrm{~s}$ per frame with the applied force of $+1.25 \mathrm{nN}$. The topographic image shows many rounded structures (presumably dust) with diameter of 10-300 nm, which had accumulated on the grating structure during the long storage period in air, without exhibiting any unstable features. The force image (as seen in Fig. 1(d)) is shown to remain relatively constant except when encountering areas that contain abruptly changing large structures (such as the grating steps). The observed force changes are due to the limited response time of the current force-feedback system to the sudden force changes. When we changed the applied force from positive to negative, the topographic and force images (Fig. 1(e) and 1(f) respectively) show some streaky fringe structures that flow in the scan direction. These streaky features represent moments of imaging instability after the system encountered a relatively large jump in tip-sample distance. The system is able to recover relatively quickly, depending on how far off the stable imaging condition (as 
NOTICE: this is the author's version of a work that was accepted for publication in Ultramicroscopy. Changes resulting from the publishing process, such as peer review, editing, corrections, structural formatting, and other quality control mechanisms may not be reflected in this document. Changes may have been made to this work since it was submitted for publication. A definitive version was subsequently published in Ultramicroscopy, 2012. DOI: 10.1016/j.ultramic.2012.09.012

indicated by the solid circle in the inset of Fig. 1(b)) is moved. When we changed the applied force back to the positive value, those streaky structures disappeared (as seen in Fig. 1(g) and 1(h)). The appearance of the streaky structures when imaging at the applied force of $-1.25 \mathrm{nN}$, and then the disappearance when imaging at the applied force of $+1.25 \mathrm{nN}$, confirms the hypothesis that the negative applied force would allow for transition between the two imaging states depending on tip-sample distance (as seen in Fig. 1(b)).

The grating structure then underwent a strict cleaning procedure (described in the Materials and Methods) to remove the adsorbed material layer in order to obtain a comparison with the underlying known hard hydrophilic grating structure. Force-distant graphs were compiled for the approach (Fig. 2(a)) and retraction (Fig. 2(b)) process. The data shows that on approach the minimum attractive force is $-39 \mathrm{nN}$ while on retraction the minimum attractive force is $-75.5 \mathrm{nN}$. When compared to the previous attractive forces of the unclean sample, the forces increased by a factor of more than 30 . The increase in attractive forces indicates that water meniscus formed between the hydrophilic sample surface and the tip due to the removal of the hydrophobic components observed in Fig. 1(c).

Interestingly, a periodic feature appeared between $0 \mathrm{~nm}$ and $8 \mathrm{~nm}$ during the approach process, as denoted as P1 and P2 in Fig. 2(a). The periodicity was calculated to be $\sim 3 \mathrm{~nm}$, possibly suggesting distancedependent structural transition $[12,13]$ of the water meniscus. In contrast, the periodic feature was absent in the force-distance curve on retraction. To verify the hardness of the sample, a linear curve fitting was made from -1.1 nm to $0.4 \mathrm{~nm}$ tip-sample distance in Fig. 2(b), thus giving a spring constant of $\sim 57 \mathrm{~N} / \mathrm{m}$. Therefore, the spring constant is shown to be much larger than the $0.77 \mathrm{~N} / \mathrm{m}$ observed in Fig. 1(b). Additionally, because a much larger negative force of $\sim 70 \mathrm{nN}$ than the $\sim 0.75 \mathrm{nN}$ of Fig. 1(b) is needed to make the transition between the two imaging conditions (as seen in Fig. 2(b)), even when the distance between the stable and unstable imaging states is only $\sim 2 \mathrm{~nm}$, it can be anticipated that the probability of reaching the unstable imaging condition is minute with an applied force of $-1.25 \mathrm{nN}$. To test this hypothesis and to verify the sample's cleanliness, a topographic image was taken with a scan area of $8 \mu \mathrm{m}$ 
NOTICE: this is the author's version of a work that was accepted for publication in Ultramicroscopy. Changes resulting from the publishing process, such as peer review, editing, corrections, structural formatting, and other quality control mechanisms may not be reflected in this document. Changes may have been made to this work since it was submitted for publication. A definitive version was subsequently published in Ultramicroscopy, 2012. DOI: 10.1016/j.ultramic.2012.09.012

$\mathrm{x} 8 \mu \mathrm{m}$, at the same applied force value of $-1.25 \mathrm{nN}$, and at the same high imaging rate of $0.6 \mathrm{~s}$ per frame.

The high-speed topographic image in Fig. 2(c) does not show the same features related to the hydrocarbon film observed in Fig. 1(c). This result verifies the samples cleanliness and indicates that the hypothesis is valid in that the system under the current circumstance did not reach the unstable imaging condition even when encountering the large topographic step heights of the grating sample. This result suggests that the imaging stability is dependent on the strength of adhesion between the tip and the surface.

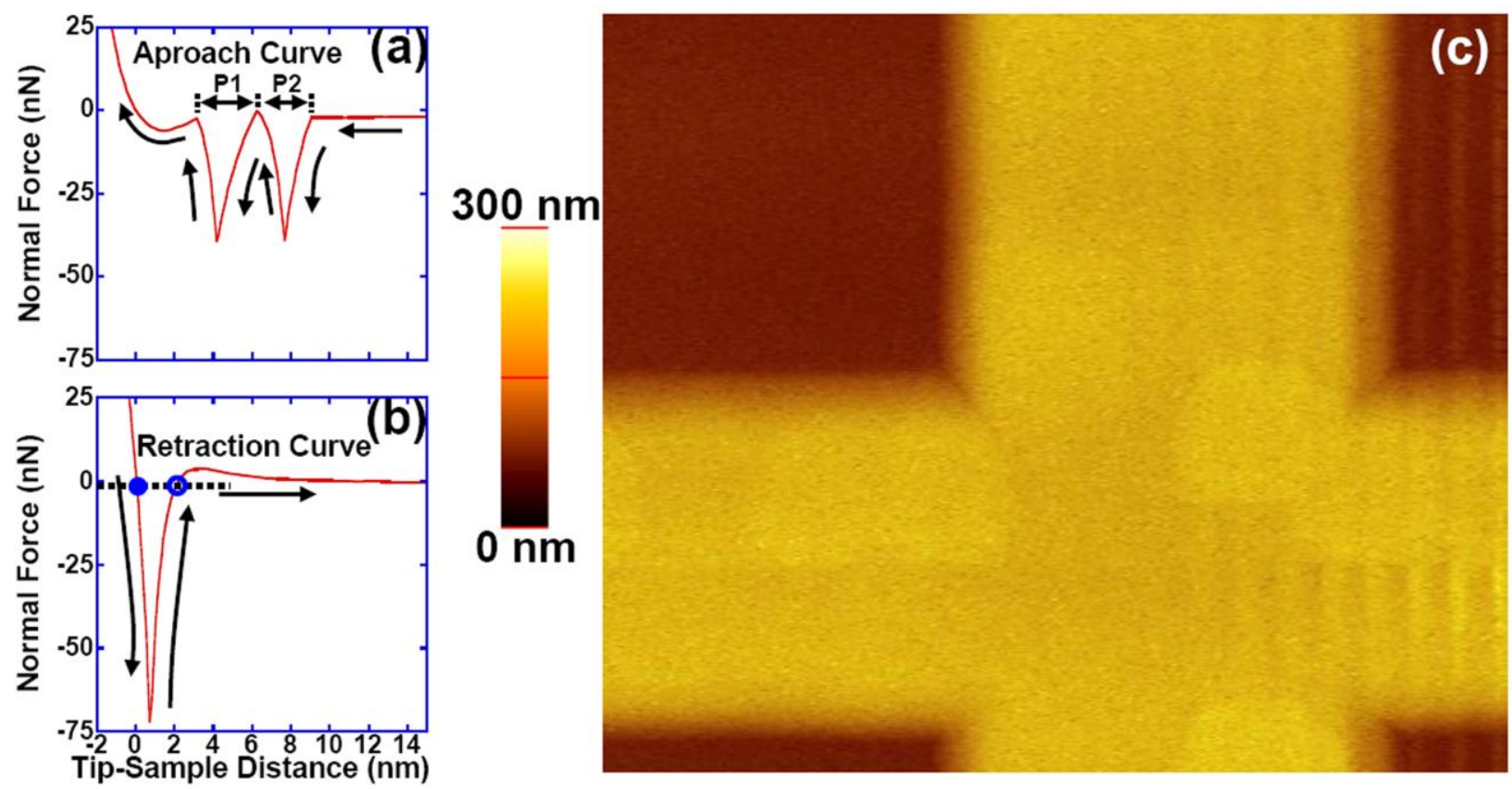

Fig. 2. Force-distance curves measured without lateral modulation at RH $30 \%$ during approach (a) and retraction (b). P1 and P2 represent the period of oscillation. The segment after P2 is the transitional interval from oscillatory to repulsive force by the sample surface. The dashed line represents the imaging applied force value; where the closed circles represent intersection of the curve with negative slope and the open circle represent intersection of the curve with positive slope. (c) Fast topographical images collected at the applied force of $-1.25 \mathrm{nN}$ in a scan size of $8 \mu \mathrm{m} \times 8 \mu \mathrm{m}$ on the cleaned grating structure at an imaging rate of $0.6 \mathrm{~s}$ per frame.

Now that the effect of the adhesion on the imaging stability is known, attention is focused on the effects derived from the imaging rate. For this part of the experiment, real-world biological applications were tested by using a very soft $E$. coli sample. The force-distance approach curve (Fig. 3(a)) showed that initially the tip did not experience any force until the tip came in contact with the biomaterial. On approach, the tip experienced a sudden attractive force of $-6.5 \mathrm{nN}$ due to the tip-contact with the material 
NOTICE: this is the author's version of a work that was accepted for publication in Ultramicroscopy. Changes resulting from the publishing process, such as peer review, editing, corrections, structural formatting, and other quality control mechanisms may not be reflected in this document. Changes may have been made to this work since it was submitted for publication. A definitive version was subsequently published in Ultramicroscopy, 2012. DOI: 10.1016/j.ultramic.2012.09.012

surface (seen to be approximately $95 \mathrm{~nm}$ from the force-distance curve). The tip-force then increased linearly as the tip-substrate distance decreased, causing the tip to push against the biomaterial. When the tip retracted (Fig. 3(b)), the linear force followed the original approaching curves until the tip distance was zero, showing that the observed force is similar to a reversible process in the repulsive force regime. However, below the tip distance of zero, the adhesive force linearly continued until it reached $245 \mathrm{~nm}$. At $245 \mathrm{~nm}$, the attractive force rapidly increased toward zero and then slowed down near zero. The relatively large pull-off force $(18 \mathrm{nN})$, in comparison to the attractive force $(6.5 \mathrm{nN})$ on approach in the forcedistance curve, indicates the existence of adhesive bonding between the tip and the biomaterial. As the distance increases after the breakage of the adhesive bonding, van der Waals forces and electrostatic interactions are the only contribution to the observed attraction at the distance larger than $260 \mathrm{~nm}$. Interestingly, the linear dependence of the force on the distance suggests that the adhesive internal material behaved like a linear spring that follows Hooke's law. A linear curve fitting from $-63 \mathrm{~nm}$ to 226 nm tip-sample distance in Fig. 3(b) provides a spring constant value of $0.076 \mathrm{~N} / \mathrm{m}$, a fractionally smaller value than seen in the soft hydrocarbon film in Fig. 1(b). Because the distance is $\sim 320 \mathrm{~nm}$ apart between the two imaging conditions (represented by the open and closed circles in Fig. 3(b)) at the standard operating applied force of $0 \mathrm{nN}$, the chance that the unstable imaging condition point will be reached is highly improbable with the observed large pull-off force of $18 \mathrm{nN}$. As before, to confirm this idea, topographic and force images were collected of biofilms with a single cell of $E$. coli on the grating sample under varying imaging rates. Fig. 3(c) shows an $8 \mu \mathrm{m}$ x $8 \mu \mathrm{m}$ high resolution topographic image and Fig. 3(d) shows the corresponding force image, taken at a rate of $\sim 128$ s per frame, of what appears to be a sequence of step-like biofilm structures (with each step height of $\sim 200 \mathrm{~nm}$ ) developed during the sample preparation. The image contains a small rod shape about 2-3 $\mu \mathrm{m}$ long and about $0.5 \mu \mathrm{m}$ in diameter in the lower right corner. We considered the structure as a lone E. coli cell because the dimension and morphology is consistent with the literature values of E. coli [17]. The E. coli was shown to have the characteristic smooth texture for the cell surface with a small indentation in the center of the cell. At the applied force of $0 \mathrm{nN}$, Fig. 3(c) shows that the tip followed step-like topographic features of the biofilm 
NOTICE: this is the author's version of a work that was accepted for publication in Ultramicroscopy. Changes resulting from the publishing process, such as peer review, editing, corrections, structural formatting, and other quality control mechanisms may not be reflected in this document. Changes may have been made to this work since it was submitted for publication. A definitive version was subsequently published in Ultramicroscopy, 2012. DOI: 10.1016/j.ultramic.2012.09.012

faithfully. The force image (Fig. 3(d)) shows a minimal root mean square (RMS) value of $\sim 0.039 \mathrm{nN}$, which corresponds to the noise level of the system. This result suggests that the slow scan rate allowed for the system feedback to compensate all topographic error signals.
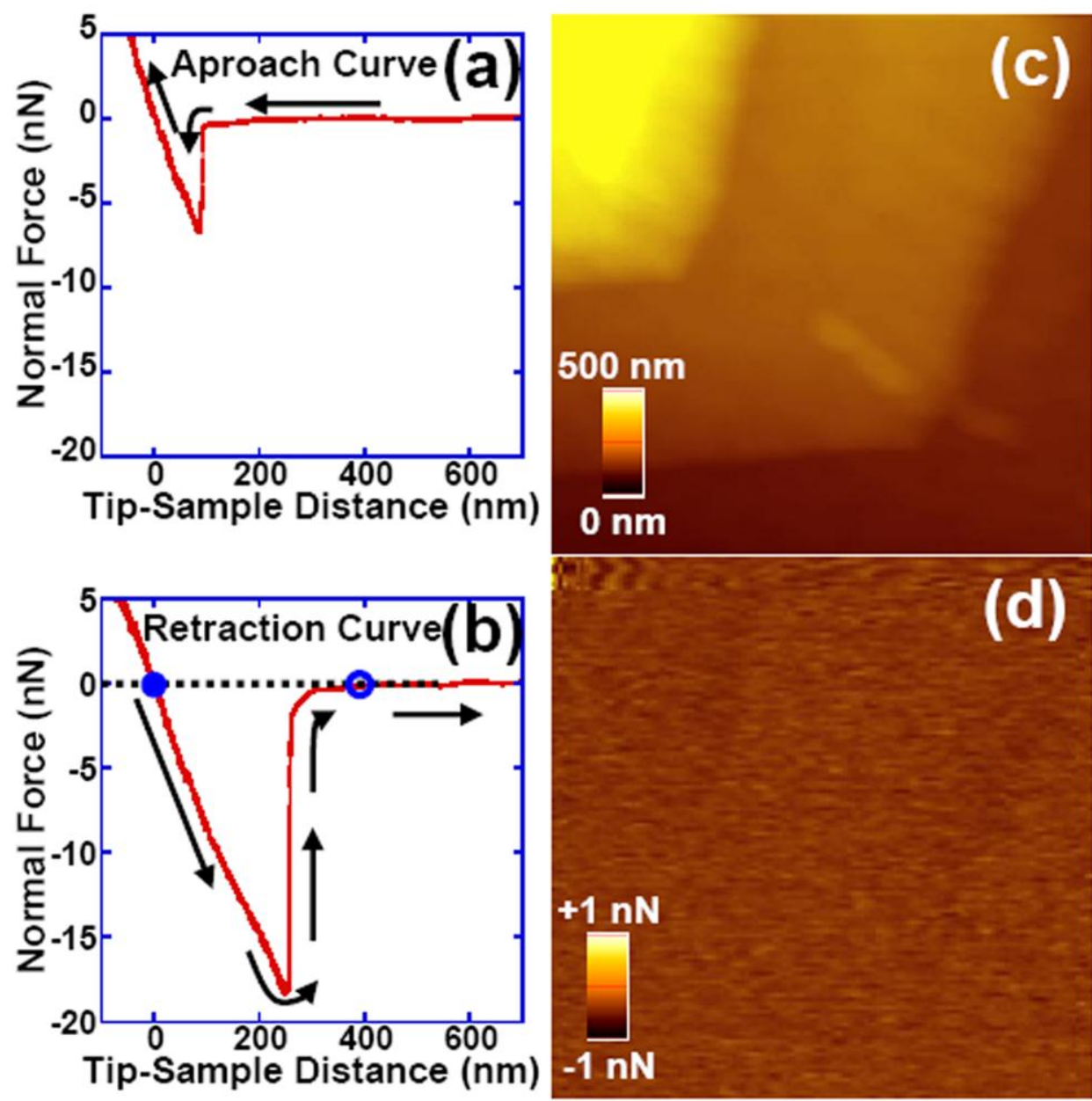

Fig. 3. Force-distance curve between the tip and an Escherichia coli sample, with a force activated voltage feedback system during approach (a) and retraction (b). The dashed line represents the imaging applied force value; where the closed circles represent intersection of the curve with negative slope $d$ the open circle represent intersection of the curve with positive slope. Topographical and force images collected simultaneously at the applied force of $0 \mathrm{nN}$ on $E$. coli biofilm with a planktonic cell at $\sim 128 \mathrm{~s}$ per frame $(\mathbf{c}, \mathbf{d})$. The scan area is $8 \mu \mathrm{m} \times 8 \mu \mathrm{m}$ with $128 \times 128$ data points.

When the imaging rate is accelerated, the force image becomes rougher arising from the limiting capability of the piezo tube's response to the rapid signal change [14]. The high resolution topographic imaging was not degraded at all as the rate was increased from $\sim 128 \mathrm{~s}$ per frame to $\sim 1 \mathrm{~s}$ per frame. Fig. 4(a) and 4(b), respectively, show the high-speed topographic and force images collected simultaneously 
NOTICE: this is the author's version of a work that was accepted for publication in Ultramicroscopy. Changes resulting from the publishing process, such as peer review, editing, corrections, structural formatting, and other quality control mechanisms may not be reflected in this document. Changes may have been made to this work since it was submitted for publication. A definitive version was subsequently published in Ultramicroscopy, 2012. DOI: 10.1016/j.ultramic.2012.09.012

at the same location at an imaging rate of $\sim 1$ second per frame. The high-speed topographic image again indicates that the tip truly follows topographic features such as steps and terraces. The high-speed force image (Fig. 4(b)), compared to the high-speed topographic image, shows better contrast in step edges and small structures on terraces attributable to abrupt signal changes. This higher contrast is due to the residual force signals that were not compensated by the topographic feedback loop when abrupt changes occurred in the sample's mechanical property or topography. As those delicate features in the high-speed force image cannot be easily identified with the topographic image only, the force image can be used as complementary information in understanding the delicate structural changes on the biological surfaces. The high-speed force images are analogous to frictional images in friction force microscopy [18, 19] where the sample's boundaries were always emphasized.

A small $y$-axis striation pattern started to appear as the rate was increased from $\sim 1$ second per frame to $\sim 0.5 \mathrm{~s}$ per frame. The pattern increased in intensity from the left to the right side in the high-speed topographic image (Fig. 4(c)) and particularly prominent in the high-speed force image (Fig. 4(d)). The fastest scan rate without observing any appreciable striation effect was approximately 1 second per frame. As the rate increased from $\sim 0.5 \mathrm{~s}$ per frame to $\sim 0.2 \mathrm{~s}$ per frame, the patterns became more dominant. The topographic (Fig. 4(e)) and force images (Fig. 4(f)) taken at the scan rate of $\sim 0.2 \mathrm{~s}$ per frame. Both images show larger striation patterns parallel to the $\mathrm{y}$-axis with $\sim 0.5 \mathrm{nN}$ in $\mathrm{RMS}$ value. At the increased imaging rate of $\sim 0.1 \mathrm{~s}$ per frame, the limited capability of RHK data acquisition system did not allow us to record high-speed topographic and force images at the same time. When the topographic image was only collected at the rate of $0.1 \mathrm{~s}$ per frame (Fig. $4(\mathrm{~g})$ ), those structures of the biofilm appeared to be blurry, showing that the striation signal was too large for the system to respond. The striation pattern has been observed previously and found to be due to the resonant vibration of the piezo tube scanner (e.g. [20-24]). Therefore, the dominant factor that limits the response speed of the system at $0.1 \mathrm{~s}$ per frame was the resonance of the piezo tube [14]. 
NOTICE: this is the author's version of a work that was accepted for publication in Ultramicroscopy. Changes resulting from the publishing process, such as peer review, editing, corrections, structural formatting, and other quality control mechanisms may not be reflected in this document. Changes may have been made to this work since it was submitted for publication. A definitive version was subsequently published in Ultramicroscopy, 2012. DOI: 10.1016/j.ultramic.2012.09.012
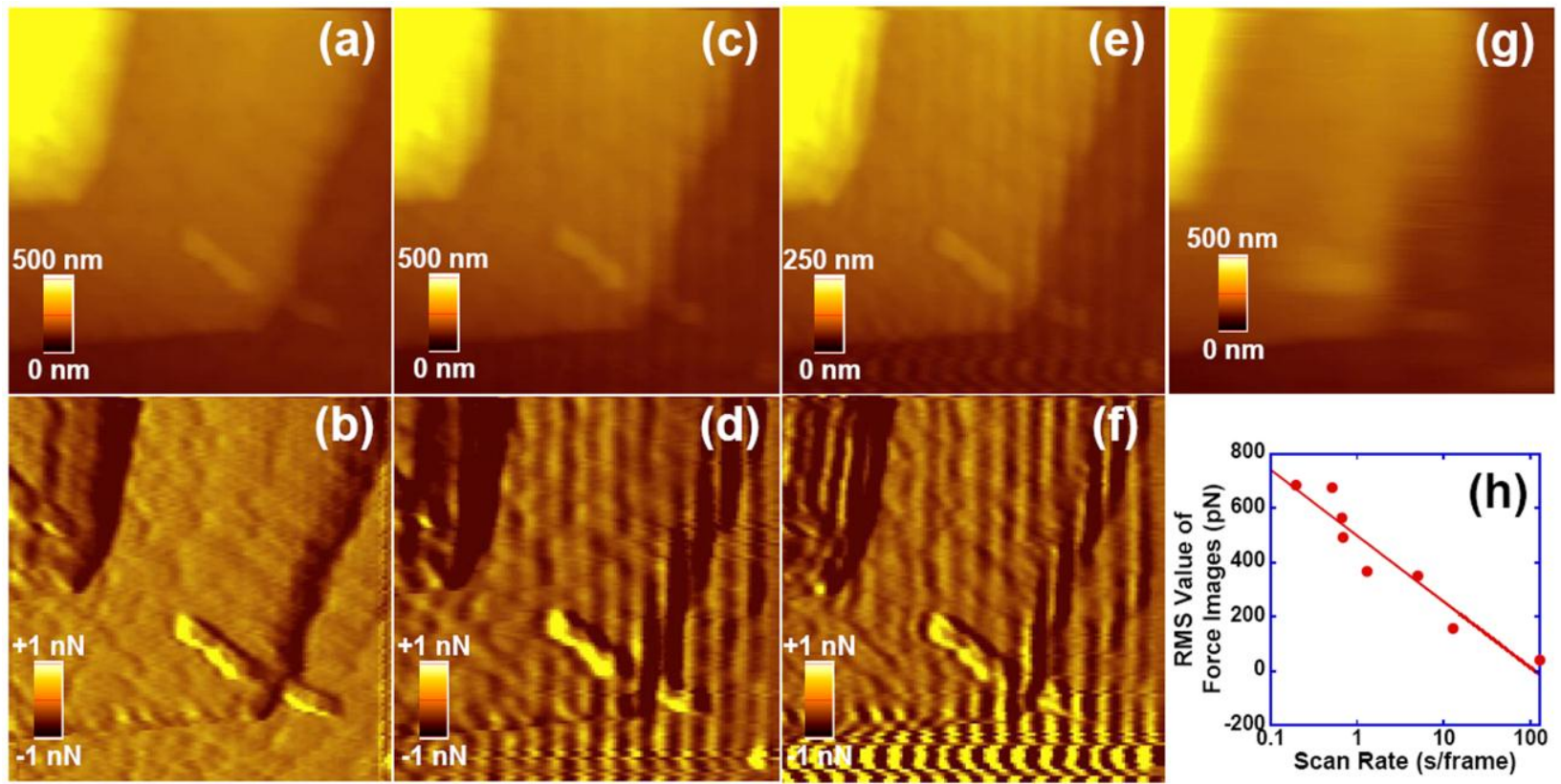

Fig. 4. Topographical and force images collected simultaneously at the applied force of $0 \mathrm{nN}$ on $E$. coli biofilm with a planktonic cell at three different scan rates: $\sim 1$ second per frame $(\mathbf{a}, \mathbf{b}), \sim 0.5 \mathrm{~s}$ per frame $(\mathbf{c}, \mathbf{d}), \sim 0.2 \mathrm{~s}$ per frame $(\mathbf{e}, \mathbf{f})$. An additional topographic image is shown for $\sim 0.1 \mathrm{~s}$ per frame (g). The scan area is $8 \mu \mathrm{m} \times 8 \mu \mathrm{m}$ with $128 \times 128$ data points. RMS value vs. scan rate plot for force images in logarithmic scale $(\mathbf{h})$.

It is important to note that, except for the striation artifacts in the series of HSAFM images, no notable changes were discernible in the $E$. coli shape and the biofilm steps. The gram-negative cell seems to have sufficient tensile strength in its cell walls that it is not damaged by the oscillatory forces even though the tip is in contact with the sample. Although, if the sample is more delicate and has less tensile strength (such as a mammalian cell), then the imaging rate will likely need to be decreased in order for the system to avoid the oscillatory force and therefore be less invasive. The RMS-force deviation of each force image is plotted as a function of the scan rate to obtain the missing force change at $0.1 \mathrm{~s}$ per frame. Interestingly, it depends on the imaging rate logarithmically as shown in Fig. 4(h). Considering the insensitivity of this logarithmic dependence, the imaging rate slight at $0.1 \mathrm{~s}$ per frame will not result in any significant force change from $\sim 0.5 \mathrm{nN}$. Also, the observed stability up to $0.1 \mathrm{~s}$ per frame indicates that the force change of $\sim 0.5 \mathrm{nN}$ was too small for the system to pass through the minimum of $-17 \mathrm{nN}$ and to attain an unstable imaging state. Therefore, the imaging rate of $0.1 \mathrm{~s}$ per frame will not cause the system to make any dramatic stable-to-unstable imaging transition. In other words, the system is able to capture 
NOTICE: this is the author's version of a work that was accepted for publication in Ultramicroscopy. Changes resulting from the publishing process, such as peer review, editing, corrections, structural formatting, and other quality control mechanisms may not be reflected in this document. Changes may have been made to this work since it was submitted for publication. A definitive version was subsequently published in Ultramicroscopy, 2012. DOI: 10.1016/j.ultramic.2012.09.012

distinguishable biological features at high-speed imaging rates up to $0.1 \mathrm{~s}$ per frame, an acquisition rate 600-1000 times faster than conventional AFMs, while maintaining a stable imaging condition [25].

\section{Conclusion}

We investigated the influence of applied loading force, hydrophilicity, and imaging rate on the imaging stability in force-feedback HSAFM. We investigated the influence by collecting force-distance curve, topographic, and force information for a variety of samples and parameters. When the force-distance curves were correlated with the images of an ambient adsorbed hydrophobic material layer on a grating sample with large step-structures, we found that stable and unstable imaging conditions depend on the distance between two points that satisfy the applied force value in the force-distance curve. When the sample adhesional property was changed from hydrophobic to hydrophilic by cleaning the surface of silicon grating, the enhanced adhesion between the tip and the surface improved the imaging stability. When we tested the influence of the imaging rate on imaging stability of an E. coli biofilm and planktonic cell, imaging was stable even at the high-speed imaging rate of $0.1 \mathrm{~s}$ per frame. Therefore, it can be concluded that the transitions between stable and unstable imaging conditions rely on applied force and hydrophilicity of the sample, but is independent of imaging rates up to the tested maximum rate of $0.1 \mathrm{~s}$ per frame. With the cooperative use of these variables that influence imaging stability, force-feedback HSAFM will potentially contribute to the imaging and understanding of hard and soft materials including biological systems.

\section{Acknowledgements:}

This research was supported by NSF DMR-1126854, NSF DBI-0852886, and the Research Corporation Single-Investigator (Cottrell College Science Award no. CC7041/7162). 
NOTICE: this is the author's version of a work that was accepted for publication in Ultramicroscopy. Changes resulting from the publishing process, such as peer review, editing, corrections, structural formatting, and other quality control mechanisms may not be reflected in this document. Changes may have been made to this work since it was submitted for publication. A definitive version was subsequently published in Ultramicroscopy, 2012. DOI: 10.1016/j.ultramic.2012.09.012

\section{REFERENCES}

1. J.P. Aimé, Z. Elkaakour, C. Odin, T. Bouhacina, D. Michel, J. Curély, A. Dautant, Comments on the use of the force mode in atomic force microscopy for polymer films, Journal of Applied Physics 76 (1994) 754-762.

2. N.A. Burnham, R.J. Colton, Measuring the nanomechanical properties and surface forces of materials using an atomic force microscope, Journal of Vacuum Science and Technology A. 7 (1989) 2906-2913.

3. K.B. Lodge, Techniques for the measurement of forces between solids, Advances in Colloid and Interface Science 19 (1983) 27-73.

4. E. Meyer, H. Heinzelmann, P. Grütter, T.H. Jung, T. Weisskopf, H.R. Hidber, R. Lapka, H. Rudin, H.J. Güntherodt, Comparative study of lithium fluoride and graphite by atomic force microscopy (AFM), Journal of Electron Microscopy—Oxford Journals 152 (1988) 269-280.

5. A. Kühle, A.H. Sørensen, J. Bohr, Role of attractive forces in tapping tip force microscopy, Journal of Applied Physics 81 (1997) 6562-6569.

6. R. Garcia, A. San Paulo, Dynamics of a vibrating tip near or in intermittent contact with a surface, Physical Review B 61 (2000) R13381-R13384.

7. T. Henze, K. Schröter, T. Thurn-Albrecht, Investigation of the different stable states of the cantilever oscillation in an atomic force microscope, Nanotechnology 23 (2012) 245702.

8. S. Santos, V. Barcons, J. Font, N.H. Thomson, Bi-stability of amplitude modulation AFM in air: deterministic and stochastic outcomes for imaging biomolecular systems, Nanotechnology 21 (2010) 225710.

9. B.I. Kim, Separation of topographic features from magnetic force images using capacitive coupling effect, Review of Scientific Instruments 80 (2009) 023702. 
NOTICE: this is the author's version of a work that was accepted for publication in Ultramicroscopy. Changes resulting from the publishing process, such as peer review, editing, corrections, structural formatting, and other quality control mechanisms may not be reflected in this document. Changes may have been made to this work since it was submitted for publication. A definitive version was subsequently published in Ultramicroscopy, 2012. DOI: 10.1016/j.ultramic.2012.09.012

10. S.A. Joyce, J.E. Houston, A new force sensor incorporating force-feedback control for interfacial force microscopy, Review of Scientific Instruments 62 (1991) 710-715.

11. J.R. Bonander, B.I. Kim, Cantilever based optical interfacial force microscope, Applied Physics Letters 92 (2008) 103124.

12. B.I. Kim, J.R. Bonander, J.A. Rasmussen, Simultaneous measurement of normal and friction forces using a cantilever-based optical interfacial force microscope, Review of Scientific Instruments $82(2011) 053711$.

13. B.I. Kim, J.A. Rasmussen, E.J. Kim, Large oscillatory forces generated by interfacial water under lateral modulation between two hydrophilic surfaces, Applied Physics Letters 99 (2011) 201902.

14. B.I. Kim, R.D. Boehm, Force-feedback high-speed atomic force microscope for studying large biological systems, Micron 43 (2012) 1372-1379.

15. Veeco, Probes and Accessories, Santa Barbara, CA, 2006, pp 88.

16. B.I. Kim, R.D. Boehm, Mechanical property investigation of soft materials by cantilever-based optical interfacial force microscopy, Scanning 10.1002/sca.21036.

17. B. Alberts, A. Johnson, J. Lewis, M. Raff, K. Roberts, P. Walter, Molecular biology of the cell 4th edition, New York: Garland Science, 2002.

18. D.R. Baselt, J.D. Baldeschwieler, Imaging spectroscopy with the atomic force microscope, Journal of Applied Physics 76 (1994) 33-38.

19. R.M. Overney, T. Bonner, E. Meyer, M. Ruöetschi, R. Luöthi, L. Howald, J. Frommer, H.J. Guöntherodt, M. Fujihira, H. Takano, Elasticity, wear, and friction properties of thin organic films observed with atomic force microscopy Journal of Vacuum Science \& Technology 12 (1994) 1973-1976. 
NOTICE: this is the author's version of a work that was accepted for publication in Ultramicroscopy. Changes resulting from the publishing process, such as peer review, editing, corrections, structural formatting, and other quality control mechanisms may not be reflected in this document. Changes may have been made to this work since it was submitted for publication. A definitive version was subsequently published in Ultramicroscopy, 2012. DOI: 10.1016/j.ultramic.2012.09.012

20. T. Ando, T. Kodera, E. Takai, D. Maruyama, K. Saito, A. Toda, A high-speed atomic force microscope for studying biological macromolecules, Proceedings of the National Academy of Sciences of the United States of America 98 (2001) 12468-12472.

21. T. Ando, T. Uchihashi, T. Fukuma, High-speed atomic force microscopy for nano-visualization of dynamic biomolecular process, , Progress in Surface Science 83 (2008) 337-437.

22. A. Miyagi, Y., Tsunaka, T. Uchihashi, K. Mayanagi, S. Hirose, K. Morikawa, T. Ando, Visualization of intrinsically disordered regions of proteins by high-speed atomic force microscopy, Chemistry Physics Chemistry 9 (2008) 1859-1866.

23. G. Schitter, P. Menold, H. Knapp, F. Allgöwer, A. Stemmer, High performance feedback for fast scanning atomic force microscopes, , Review of Scientific Instruments 72 (2001) 3320-3931.

24. A.W. Sparks, S.R. Manalis, Scanning probe microscopy with inherent disturbance suppression, , Applied Physics Letters 85 (2004 ) 3929-3931.

25. B.I. Kim, R. Clark, T. Clark, Long-term structural changes of plasmid DNA studied by atomic force microscopy, Scanning 33 (2011) 408-412. 